
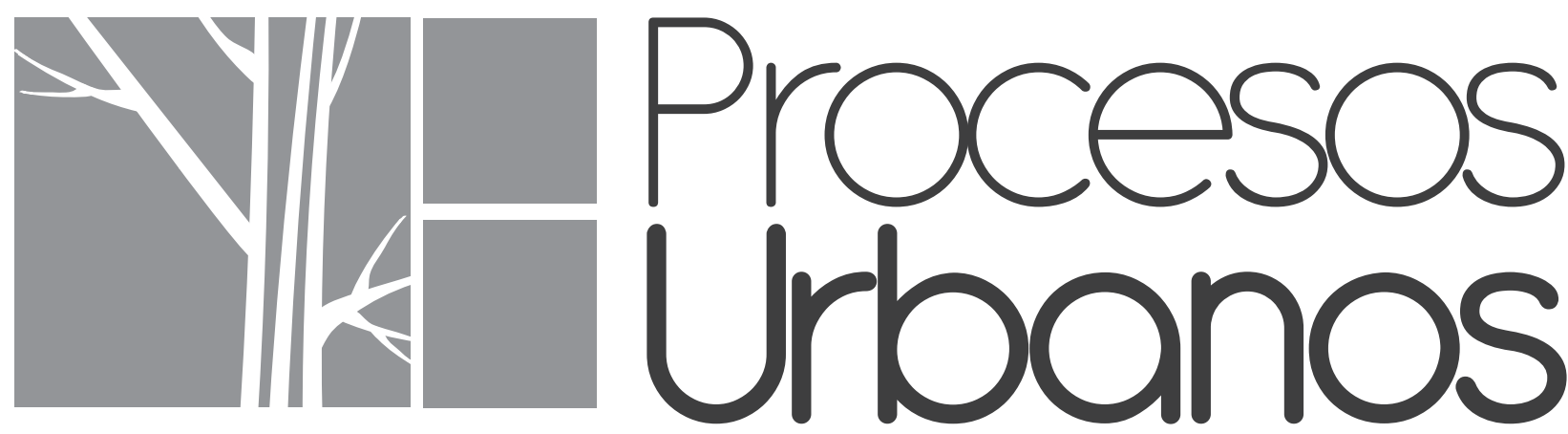

Revista de Divulgación Científica 


\section{Revista Procesos Urbanos}

Facultad de Ciencias Básicas, Ingeniería y Arquitectura.

ISSN: 2422-085X

Sincelejo, Enero - Diciembre de 2014

Piedad Martínez Carazo

Rectora

\section{Lidia Flórez de Albis}

Vicerrectora Académica

\section{Jhon Víctor Vidal}

Director de Investigaciones

\section{Pedro Arturo Martínez Osorio}

Editor

\section{Comité Editorial:}

PhD. Alexander Niño Soto. Universidad Antonio Nariño

PhD. Jorge Ramírez Nieto. Universidad Nacional de Colombia

Msc. Juan Carlos Pergolis. Universidad Católica de Colombia

PhD. Piedad Martínez Carazo. Corporación Universitaria del Caribe, CECAR

PhD. Emiro F. Martínez. Universidad de York, Canadá

\section{Comité Científico:}

Msc. Gloria Aponte García. UPB, Medellín

Msc. Jorge Vásquez Muñoz. UPB, Medellín

PhD. Jorge Gómez Ricardo, Universidad del Magdalena

\section{Editorial CECAR}

\section{Libia Narváez Barbosa}

Coordinadora Editorial

\section{Eduardo Támara Galván}

Corrector de Estilo

\section{Alejandrina Jaramillo y Roger Goez Castillo}

Diseño Gráfico

Diagramación e Impresión:GRÁFICAS DEL CARIBE S.A.S.

Cra. 1B No. 40-42 Montería Tel. (57) (4) 7826622 Telefax (57) (4) 7817112

Email: diseno@graficaribe.co

\section{Dirección:}

Carretera Troncal de Occidente

Kilómetro 1 Vía a Corozal

Tel: 280402928040172804018 Ext 1115

www.cecar.edu.co

http://cecar.edu.co/revista-procesos-urbanos 


\title{
HÁBITAT $Y$ SOSTENIBILIDAD DE LOS REASENTAMIENTOS HUMANOS EN CONDICIÓN DE MARGINALIDAD ${ }^{1}$
}

\author{
Habitat and sustainability of human condition on resettlement marginality
}

Gloria Zuleta Roa

Fecha: Recibido febrero 10 de 2014 / Aceptado abril 28 de 2014

\section{RESUMEN}

Elhábitat, lugardondeseredefineterritorialmente la cultura de un grupo diverso, es un escenario para los encuentros y desencuentros, la luz y la oscuridad. La investigación se origina en los procesos de reasentamiento habitacional que ocurrieron en el eje cafetero a raíz del terremoto del año 1991, y tomó como estudio de caso específico la Ciudadela Simón Bolívar en Armenia. Su objetivo, indagar posibles causas de la fuerte ruptura que se presentó en el proceso de reasentamiento. El método de trabajo, se desarrolla mediante una investigación - acción, buscando reconstruir una experiencia replicable en otros sectores del país. Como resultados del estudio se proponen una serie de factores que deben tenerse en cuenta para el desarrollo de una estrategia habitacional sustentable para poblaciones en condición de vulnerabilidad y de marginalidad. Entre las conclusiones más significativas se plantea, tener siempre presente en los procesos de intervención del hábitat,que se construye y se edifica para seres humanos que en su complejidad no son sujetos de diagnósticos irrefutables, lo planeado no siempre es el resultado de lo esperado. Siendo necesaria la modelación conjunta de las estrategias de sostenibilidad, superando las visiones cortoplacistas que solo llevan a una pérdida enorme de recursos, de esfuerzos y de agotamiento del capital social que se haya logrado construir.

\author{
Palabras Clave: Hábitat, asentamientos \\ humanos, marginalidad, sostenibilidad, \\ desarrollo humano.
}

\begin{abstract}
The habitat, place where there is territorially re-defined the culture of a diverse group, is a scene for the meetings and misunderstandings, the light and the darkness. The origins of the research in the processes of housing resettlements that happened in the coffee axis immediately after the earthquake of the year 1991, and take Simon Bolívar neighborhood in Armenia, as a study case of specific case. His aim was to investigate possible reasons of the strong break that one presented in the process of resettlement. The method of work - action develops by means of an investigation, seeking to reconstruct an experience replicable in other sectors of the country. The results of the study propose themselves a series of factors that must be born in mind for the development of a sustainably strategy for populations in condition of vulnerability and of marginality. The most significant conclusions appears, to have always present in the processes of intervention of the habitat, which is constructed and is built for human beings who in his complexity are not subjects of irrefutable diagnoses, not always it is the result of the awaited thing. Being necessary the modeling combines of the strategies of
\end{abstract}

\footnotetext{
${ }^{1}$ Investigación hecha para optar el título de Magister en Hábitat Línea de estudios en vivienda de la Universidad Nacional de Colombia, sede Bogotá.

Economista, Universidad Autónoma de Colombia. Septiembre 25 de 1987. Especialización en Gerencia de Proyectos Educativos, Universidad Cooperativa de Colombia, con énfasis en el uso de las tecnologías informáticas de comunicación. Especialización en Construcción Sostenible. Universidad Colegio Mayor de Cundinamarca. Maestría en Hábitat-Estudios en Vivienda de la Universidad Nacional de Colombia - sede Bogotá. Email gloriaizuleta@gmail.com. Docente catedrática de la Institución Universitaria Colegio Mayor de Antioquia y consultora en temas de hábitat y medio ambiente.
} 
sustainability, overcoming the short visions that only lead to an enormous loss of resources, of efforts and of weakening of the share capital that has been achieved to construct.

Keywords: Habitat, human settlements, marginality, sustainability, human development.

\section{INTRODUCCIÓN}

Repensar en el presente, seis años después de la primera presentación de la investigación acerca de las problemáticas que se presentan sobre los reasentamientos humanos en condiciones de marginalidad, bajo el enfoque de desarrollo sostenible, libertad, capacidades humanas y desarrollo humano, implica hacer una mirada ambientalista desde la complejidad, con la cual se busca integrar la conceptualización institucional del desarrollo sostenible con la visión ambiental compleja que insta a mirar, de forma amplia e incluyente, los procesos sociales, económicos, culturales y su interrelación con el medio natural y construido. Así, a manera de epígrafe se presenta un bello texto de Estanislao Zuleta, que busca presentar la reflexión como un aporte a las muchas lecturas que se hacen de estos procesos, más allá de pretender que sea la verdad revelada o la solución mesiánica a las problemáticas expuestas,

La mayor dificultad consiste en decir lo que ya se sabe, en reconocer lo que secretamente se conoce; que es un abismo aterrador porque se conoce, porque si no se conociera sería una palabra vacía; pero si se reconoce nos hace pedazos. Aquí encontramos el vínculo entre 10 "que ya se sabe", y la exigencia de valor, de audacia y de arriesgarse a ser descubridor. (Zuleta. E, 1982. P.25).

\section{METODOLOGÍA}

La investigación se origina en los procesos de reasentamiento habitacional que ocurrieron en el eje cafetero a raíz del terremoto del año 1991, y tomó como estudio de caso específico la Ciudadela Simón Bolívar en Armenia. En el proceso de reconstrucción 56 Procesos a través de la llamada vitrina inmobiliaria, constructoras independientes, constructoras institucionales y OPV -Organizaciones Populares de Vivienda- mostraban a los damnificados y posibles beneficiarios de soluciones de vivienda, distintas alternativas, no solo en lo referido al diseño arquitectónico, sino a las posibilidades de acceso a las mismas a partir de los subsidios que el Estado otorgaba a las familias. En este proceso la OPV Simón Bolívar, presentó una propuesta que llegó a ser hito en el proceso de reconstrucción por fundamentarse, desde el discurso, en procesos de sostenibilidad que incluían la construcción de comunidad, de capital social a la par de la construcción física de las viviendas. La OPV organizó escuela de líderes, buscó involucrar a los jóvenes como estrategia para prevenir futuros problemas delincuenciales asociados con el consumo de psicoactivos, muy comunes en la región y en la época; los equipamientos fueron construidos con los mejores materiales e incluso se construyeron escuela y colegio como parte de la estrategia de construcción de capital social. Sin embargo, en algún momento del proceso se presentó una fuerte ruptura de la propuesta inicial; gran parte de los equipamientos fueron destruidos por algunos habitantes del barrio y este se convirtió, como muchos otros, en el proceso de reconstrucción en hábitat del miedo, lugares donde ni el servicio público de taxis entraba.

De allí nace la inquietud por indagar las posibles causas, por encontrar el punto de quiebre del proceso que, desde lo planeado, se constituía en una experiencia que podía ser perfectamente replicable en otros sectores del país. Para entrar al barrio se contactaron algunos de los líderes con los cuales se hicieron largas entrevistas, se hicieron registros fotográficos y recorridos en las horas en las que era posible vivir-andar el barrio. El trabajo de campo se llevó a cabo porque gran parte de la comunidad seguía creyendo en el proceso, especialmente un grupo de mujeres cabeza de familia que había estado desde los comienzos de las escuelas. Lo demás es escribir, contar desde el alma y desde la reflexión académica los encuentros y desencuentros entre teoría y práctica... No es recomendable, en ningún 
proceso, olvidar que en la condición humana perviven la luz y la oscuridad, y hay que tener especial cuidado en los agentes culturales, económicos y sociales que pueden hacer detonar una u otra.

\section{RESULTADOS}

Se propone inicialmente categorizar el hábitat humano en tres conceptos esenciales: Sociedad individuo y medio ambiente. De estas tres categorías, dos se recrean permanentemente y en continua interacción en el desarrollo de la ponencia: la sociedad y los individuos. Así, de una parte, la definición del hábitat que plantea Leff (1998: 240) es el fundamento para reflexionar sobre el diseño sustentable. El hábitat, como el lugar donde se redefine territorialmente la cultura de un grupo diverso, heterogéneo como son los seres humanos que se asocian alrededor de una necesidad común: la vivienda, un lugar donde habitar un espacio propio, donde se halla un nuevo significado de vivir, unas nuevas prácticas sociales que retoman a la persona en sus dimensiones física, cognitiva y emotiva.

Pero no siempre coincide lo soñado y proyectado con el resultado final; más aun cuando los procesos de reasentamientos, fundamentados en la construcción de vivienda y de unas nuevas prácticas socio-económicas, están íntimamente relacionados con los modos de habitar, de ser social y culturalmente, y tal como lo afirma Touraine, "ningún país, ninguna institución, ningún individuo tiene, por sus éxitos pasados, la posibilidad de comprender y dominar las nuevas formas de vida personal y colectiva, (1999: 23).

De esta manera se religan los conceptos de hábitat y desarrollo y su relación con los individuos a partir del planteamiento de Amartya Sen, en Desarrollo y libertad, cuando afirma que el desarrollo es un proceso de expansión de las libertades reales de que disfrutan los individuos (2003:19), libertades que se ven constreñidas por la pobreza e inequidad y una marcada ausencia de capacidades humanas, relacionadas con la falta de oportunidades sociales y económicas que permitan el acceso a la satisfacción de las necesidades básicas y más allá, al desarrollo de los individuos y del tejido social que facilite a un grupo social fortalecerse y convertirse en una comunidad activa y protagonista de su futuro.

Esa ausencia de capacidades y de la libertad para participar activamente en las decisiones que afectan el entorno inmediato de las personas y que permiten la sostenibilidad de los procesos sociales, económicos, ambientales que se han hecho evidentes en las estrategias de reconstrucción y manejo de los asentamientos humanos, bien sea por desastres de origen natural o antrópico. Esa situación va más allá de la estrechez de recursos o la falta de capital, y se relaciona más con la inequidad, la concentración de la riqueza y la ausencia de voluntad política por parte de las instituciones y el Estado en general, orientada a propiciar el entramado social, el fortalecimiento de las organizaciones comunitarias, pues con suficientes oportunidades sociales, los individuos pueden configurar en realidad su propio destino y ayudarse mutuamente. (Sen, 2003:28).

El desarrollo, visto así, trasciende el simple crecimiento económico de una región si lo que se pretende es ir más allá de lo físico, de lo urbano y formularlo en términos de desarrollo humano. Implica una revisión de las estructuras macroeconómicas y el establecimiento de políticas a nivel nacional que integren las propuestas del modelo planteado, es decir que inserten la región en el contexto nacional, bajo las premisa de un accionar más eficaz del Estado, sin que se caiga en una ampliación de la burocracia y actitudes paternalistas que, en últimas, colocan a la comunidades altamente vulnerables social y económicamente, en condiciones de minusvalía para agenciar su propicio desarrollo, y de una participación social efectiva, que involucre a todos los agentes sociales: Estado, comunidad, empresa privada.

Así se entrelaza el desarrollo con la libertad y las capacidades humanas, a partir de la posibilidad que tienen las personas de lograr fuentes de trabajo, servicios de salud, educación, recreación, factores que influyen directamente en las libertades que tienen los individuos. Libertades referidas a la posibilidad de participar activamente de los 
servicios económicos, del quehacer político, de las oportunidades sociales y con ello, de las garantías protectoras alrededor del desarrollo como persona, como ser humano en interacción armónica con el medio natural.

Los fines y los medios del desarrollo obligan a colocar la perspectiva de la libertad en el centro del escenario. En este enfoque, los individuos han de verse como seres que participan activamente -si se les da la oportunidaden la configuración de su propio destino, no como meros receptores pasivos de los frutos de ingeniosos programas de desarrollo. (2003-75)

Enfoque que difiere profundamente de la concepción economicista, fundamentada en el crecimiento de la riqueza, independiente de los efectos devastadores que este crecimiento pueda tener sobre los individuos y sobre el medio ambiente. En la medida en que la expectativa por una mejor calidad de vida se concentra en el acceso a la riqueza, independientemente de la validez de los medios para alcanzarla, se resquebraja el tejido social que se haya logrado construir, se profundiza la desigualdad y las estrategias de desarrollo en que se fundamentan el modelo propuesto para la reconstrucción, se quedan en los documentos escritos, sin lograr concretarlas en acciones reales que aumenten las capacidades de las personas. Se requiere por lo tanto, un replanteamiento de lo económico aunado a una política social que privilegie las posibilidades de participación de la comunidad.

En el mismo sentido se expresa Klisksberg, al afirmar que la política social se construye en uno de los factores estratégicos del desarrollo humano, pues en la medida en que la comunidad tenga conciencia del rol que ejerce y haya una gerencia efectiva a partir de unas políticas adecuadas, la contribución de las personas y del colectivo social mismo, es fundamental, y en sentido contrario, si se trabaja sobre políticas débiles y aisladas, el deterioro social puede ser aun mayor. (2003: 23).

De otro lado está la conceptualización sobre el desarrollo sostenible y su contraposición frente al concepto de sustentabilidad. Se parte de la propuesta de Manfred Max-Neef, quien desde una visión compleja, propone centrar los discursos, no desde enfoques netamente económicos, cual es el paradigma de desarrollo sostenible, sino desde el ser humano, sus necesidades y la satisfacción de las mismas. El autor es enfático en decir que un nuevo enfoque "no puede reducirse a meros arreglos cosméticos de un paradigma en crisis" (1997: 27) y que parte de miradas arcaicas centradas en el crecimiento económico y no desde el ser humano. Es desde esta situación que se propone el desarrollo a escala humana referido a las personas y no a los objetos, como lo destaca Max-Neef. Así, desde las necesidades humanas y su satisfacción se llega a una lectura de lo que se denomina calidad de vida en consonancia con la apropiación del territorio en la construcción de sentido del habitar.

Respecto al desarrollo sostenible, es conveniente remontarse al nacimiento de este concepto, el cual se formuló desde el informe Bruntland, en la Comisión de las Naciones Unidas, en 1987, según el cual "el crecimiento económico, a largo plazo sólo podrá garantizarse si se tienen en cuenta sus repercusiones ambientales y si en el consumo de recursos naturales no se hipotecan las necesidades de generaciones futuras. (Albet, 2000: 67-6). Vale la pena aclarar cómo el término se generó desde ámbitos meramente económicos, con lo cual las implicaciones desde la complejidad, cual es la realidad, obligan a mirar con detenimiento una propuesta que, aparentemente busca armonías globales para con el planeta, pero desentendiéndose de las interacciones sociales y culturales. Al respecto, comenta Roberto Fernández, cómo esta propuesta constituye una "exposición conceptualnormativa acerca del estado que los países hegemónicos confieren a la temática ambiental y a sus prioridades, en torno a la sustentabilidad de una clase de desarrollo que se reconoce adscrito a la economía de mercado". (Fernández, 2000: 58).

Por otra parte, sobre el concepto de barrio, Michel de Certeau, en su documento La invención de lo cotidiano (1999: 68) hace una aproximación hacia ese ámbito 
denominado barrio. Expresa el autor: El barrio puede entonces entenderse como esa porción conocida del espacio público en general, donde se insinúa poco a poco un espacio privado particularizado debido al uso práctico cotidiano de ese espacio. La fijeza del hábitat de los usuarios, la costumbre recíproca derivada de la vecindad, los procesos de reconocimiento de identificación- que ocupan su sitio gracias la proximidad, a la coexistencia concreta sobre un mismo territorio urbano. Desde un panorama tanto exploratorio en trabajo de campo, como desde la conceptualización, este autor propone la idea de barrio como el lugar donde se manifiesta la coexistencia con los vecinos, a los que liga la ritualización de la cotidianidad en ámbitos del reconocimiento.

Asimismo, el barrio se constituye, desde el espacio público, como extensión inmediata del mundo privado, la casa, y filtro hacia el aparentemente ilimitado espacio de la ciudad. Es por esto, que el barrio es un lugar de repliegue, donde los vecinos se reconocen y manejan una serie de códigos, convenciones y rituales que escapan a la simple vista de un foráneo. Este sistema de signos, expresado en el ámbito público, la calle, es lo que determina la identidad del barrio y se construye día a día. Así, los procesos de reconocimiento, la familiaridad, los usos y los ritos de vecindad constituyen parte de esa pléyade de significados que enmarca la esencia de un barrio.

El mismo Certeau, pone de manifiesto cómo en esa vida colectiva, el grupo se sobrepone al individuo en la medida que este debe replegarse frente a los intereses del grupo, como parte de un "contrato implícito", donde la conveniencia es parte del beneficio obtenido por vivir en esa comunidad. Igualmente se hace énfasis en determinar cómo el barrio es el espacio de una relación con el Otro, como ser social. Esta noción de individuo se emparenta con la propuesta de Elina Dabas para quien el sujeto no es únicamente la dimensión biológica, sino la construcción desde el intercambio en un mundo complejo desde ámbitos de la sociabilidad, donde a "través de los vínculos sociales de afecto, de lenguaje, de comportamientos, el sujeto se va autoorganizando. (Dabas, 2002: 66). Así la vida cotidiana es un acto cultural, es decir, no responde al azar, con lo cual cada individuo forma parte de una red de signos sociales cuya misma existencia es incluso anterior a él en la medida en que el barrio constituye una construcción colectiva. Dice al respecto Certeau:

La relación entrada/salida, dentro/fuera, confirma otras relaciones (domicilio) trabajo, conocido/desconocido, calor/ frío, actividad/pasividad, masculino/ femenino...) siempre se trata de una relación entre sí mismo y el mundo físico y social; es la organizadora de una estructura inicial y hasta arcaica del sujeto público urbano mediante el pisoteo incansable por cotidiano, que mete en un suelo determinado los gérmenes elementales de una dialéctica constitutiva de la conciencia de sí, que adquiere en ese movimiento de ir y venir, de mezcla social y repliegue íntimo, la certeza de sí misma como algo inmediatamente social. (2005: 25.)

Igualmente, se pone de manifiesto, cómo el barrio, con su universo significante, está presente desde la infancia y constituye toda una técnica de reconocimiento plegarse a él cuando se llega de improviso. Por otra parte, Jane Jacobs expresa, quizá desde una óptica un tanto romántica, cómo la esencia del barrio y sus calles se han perdido con las nuevas propuestas urbanísticas que desconocen precisamente la dialéctica del vecindario: "la ciudad reconstruida ha hecho trizas una función básica de las calles de una ciudad y, al hacerlo, ha liquidado necesariamente su libertad". (1967: 24). Con estas palabras la autora recurre al reconocimiento de la calidad tradicional del barrio con sus calles protegidas por miles de ojos, que interactúan: los vecinos quienes ritualizan ballets cotidianos de proxemia y comunicación. Asimismo nuevamente enfatiza cómo el salir a la calle es un acto público y por ende cultural y, el individuo en esa escena de contactos que es el espacio público del barrio, participa de una red y un tejido de respeto mutuo y aceptación implícita de los códigos colectivos solamente reconocidos por los vecinos. Añade, cómo esa una aceptación y construcción de confianza y respetos difícilmente pueden ser estandarizados e institucionalizados, ya que surgen de la simple convivencia y reconocimiento. 
En este sentido, el barrio, entendido como espacio público, se constituye como lugar de la acción, así como de los procesos de identificación comunitarios y de familiaridad. (Josepf, 1999: 10). Así, incluso el espacio adquiere carácter político en la medida que constituye terreno de formas funcionales y de vínculos sociales alrededor de problemas y situaciones comunes. En esa construcción de identidad que evidencia eso que se denomina barrio, surge una relación entre los vínculos sociales y el territorio, como espacio que adquiere significado como lugar precisamente de la fusión de los símbolos con la geografía. Al respecto, vale la pena tener en cuenta la reflexión de Maurice Halbwachs, quien desde terrenos de la memoria colectiva, manifiesta cómo algunos barrios, sobre todo los tradicionales con gran carga histórica, o los aislados de las dinámicas del centro de las ciudades, constituyen universos cerrados donde las relaciones son expresivas y la situación de vecindad se multiplica. Asimismo comenta cómo la relación del grupo social se mueve con mayor dinamismo que el espacio y, la memoria colectiva crea imágenes que por superposición de capas, cual es la cultura, otorga gran fuerza a las redes, vínculos y universo de símbolos elaborados en complejos procesos históricos. Asimismo manifiesta cómo, en el terreno de lo físico, se genera una larga duración, en términos históricos, que otorga solidez al espacio y, un sentido de arraigo y estabilidad al habitante, quien se acostumbra a su lugar reconocido y seguro. Dice Halbwachs, (2004: 136):

Cuando un grupo humano vive durante mucho tiempo en un lugar adaptado a sus costumbres, no sólo sus movimientos, sino también sus pensamientos se regulan según la sucesión de imágenes materiales que le ofrecen los objetos exteriores. Ya se pueden suprimir en parte o modificar la dirección, la orientación, la forma o el aspecto de estas casas, estas calles, estos pasos, o cambiar solamente el lugar que ocupan uno respecto de otro. Las piedras y los materiales no resistirán. Pero los grupos se resistirán $y$, en ellos se enfrentarían, no tanto el apego a las piedras, como el que tienen a sus antiguos lazos.
Según esta reflexión, no es en sí el hecho físico el que otorga identidad y refugio al grupo humano, sino la interrelación entre el colectivo y el territorio, con lo cual, las marcas del lugar, los aspectos estéticos, los materiales, las formas, otorgan a través de la cotidianidad y de la red simbólica construida colectivamente en capas sucesivas, significados al espacio. Bien dice Halbwachs cómo desde esa compleja red surge un apego del grupo a su lugar $y$, se resiste a ver desaparecer o cambiar elementos físicos de ese territorio y, cuando eso ocurre siente que parte de sí mismo se ha ido con ellos. Incluso, dice Halbwachs, es tan grande el apego del colectivo a su entorno, que a veces, cuando las circunstancias del lugar cambian buscan hallar su antiguo equilibrio en nuevas circunstancias. Obviamente, esto se logra cuando existe una fuerte cohesión colectiva que se sobrepone al entorno. El mismo autor reitera que los grupos están unidos a un lugar, pues al compartir un territorio, se generan lazos sociales. Asimismo manifiesta, cómo quizá esta es una de las únicas formas de organización que nace del compartir un espacio, en un mundo donde precisamente las demás formas lo que hacen es separar y desligar.

Ahora bien, en una mirada desde la complejidad y frente a las dinámicas que enfrentan las ciudades hoy, el barrio adquiere otra dimensión en la medida que desde su planificación y administración debe asegurársele ser partícipe de los procesos culturales, económicos y sociales de su entorno, y no a estar condenado a depender para lograr una subsistencia. Es así cómo desde una consideración amplia de ciudad desde la noción y la gestión, Jordi Borja, (2003: 125), propone una serie de mandamientos a tener en cuenta para asegurar inclusión y calidad, de vida a las diferentes comunidades de una ciudad. Se parte de la consideración de la ciudad en sus espacios públicos como la esencia de su existir, donde se rescate la dimensión sociocultural como lugar de relación y de la identificación, así como de contacto entre las personas y lugar para la expresión comunitaria.

Se ofrece una mirada hacia la potencialidad del espacio en la identificación simbólica, la construcción de imaginarios y la construcción de redes. Como contraposición, 
el aludido autor denuncia cómo desde las políticas públicas de reasentamientos, con programas monofuncionales y sectorizados, se generan "grupos de la ciudad que se degradan rápidamente, por falta de inserción urbana, por anomía sociocultural, o pobreza de equipamientos", entre otros factores. Cuestiones como la defensa del espacio público, el acceso a las centralidades ya existentes en la ciudad, el derecho a disfrutar y vivir las monumentalidades y la memoria, tanto institucional como popular, constituyen parte de lo que Borja denomina como el derecho a la ciudad, que toda comunidad debe tener, más aun, aquellas que por desastres de origen natural o antrópico, han sido desplazadas de su hábitat cotidiano.

Cualquier tipo de exclusión, territorial, social, cultural, de género, de edad..., se debe erradicar de cualquier propuesta en materia de asentamientos sostenibles $y$, es deber el

"desenclavar los ghettos, legalizar y regenerar el hábitat marginal, promover la cooperación de los habitantes en la cualificación de los espacios públicos y mejora de la vivienda, introducir la mixtura social, localizar equipamientos $y$ elementos monumentales que proporcionen visibilidad y dignidad a la zona". (200: 125)

Desde ámbitos culturales, se integra a lo anterior el favorecimiento de integración comunitaria e identidad. Desde estadios socioeconómicos se plantea el asegurar sostenibilidad a partir de generación de empleos in situ y en cadenas productivas, programas permanentes de educación formal y continuada, así como apoyos en fortalecimiento de la autogestión.

Es necesario correr el riesgo y atreverse a reflexionar, y a modo de conclusión, proponer algunos factores que deben tenerse en cuenta para el desarrollo de una estrategia habitacional sustentable para poblaciones en condición de vulnerabilidad y de marginalidad.

- Las estrategias formuladas por el Estado, en relación con la promoción de un hábitat sostenible para las poblaciones en condiciones de marginalidad, requieren más allá de una política de vivienda el fortalecimiento del capital social y la reactivación económica de la región donde se pretende implementar las estrategias.

- La participación activa de los damnificados que no son propietarios de vivienda y que hacen parte de la marginalidad en la ciudad, se convierte en elemento esencial para acceder a las soluciones de vivienda propia.

- Es necesario que los agentes sociales generen mecanismos que permitan una participación verdadera, una participación que implique incidir directamente en los escenarios de toma de decisiones, al derecho de autogobernarse, que se consigue, entre otras cosas, con mayores oportunidades educativas de construir sus propios horizontes, pues la no sostenibilidad del proceso lleva a un círculo vicioso; se pierde el amor propio, se vuelve a los viejos esquemas de violencia y desarraigo y se da la excusa para que desde algunos sectores de la ciudad, se señalen estos asentamientos como focos de descomposición social.

- La ponderación de la variable vivienda propia, en muchos casos pudo tener una interpretación que no se adecua a la realidad de un amplio grupo de familias de arrendatarios, que no tienen referentes de las obligaciones y compromisos inherentes a la propiedad privada de la vivienda, convirtiéndose entonces la vivienda propia en una carga negativa de presión social dentro de la comunidad.

- Es la eterna réplica de un modo de ser, de pensar y de actuar, tanto de quienes dirigen como de los dirigidos con un esquema repetido por generaciones, como el cuento del gallo capón, en el que la historia se repite incansablemente sin hallar un fin determinado.

\section{CONCLUSIONES}

En últimas y amparándose en el derecho a la contradicción, se propone como conclusión tres estrategias encaminadas a construir un

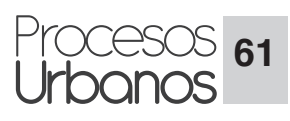


PROCESOS URBANOS - Revista de Divulgación Científica Vol. 1 Enero - Diciembre 2014 (55 - 62)

hábitat sostenible, abiertas a la discusión, sujetas a ser demolidas por los eruditos del hábitat o interiorizadas por los que aún sueñan con las utopías de un nuevo modo de vivir:

1. Tener en cuenta que el hábitat debe ser diseñado por equipos interdisciplinarios en diálogo con quienes son los actores del proceso, es importante considerar que más allá de las cuatro paredes construidas, el hábitat es también el sentido que sus habitantes le dan al espacio construido, el lugar donde el fuego está presente como un espacio de descanso y no de angustia por la sobrevivencia.

2. Recordar que se construye y se edifica para ser humanos que en su complejidad no son sujetos de diagnósticos irrefutables y que al contrario, lo planeado no siempre es el resultado de lo esperado. Así, es necesaria la modelación conjunta de las estrategias de sostenibilidad, superando las visiones cortoplacistas que en últimas solo llevan a una pérdida enorme de recursos económicos, de esfuerzos y de agotamiento del capital social que se haya logrado construir.

3. Capacidad de interacción de los agentes sociales, desde la posibilidad directa de proponer, participar ydecidir de las comunidades, en especial de las marginadas, hasta la voluntad política y económica de quienes detentan el poder, bien sea desde el Estado o de los grupos económicos que, local o globalmente determinan las pautas de crecimiento económico y tangencialmente, de desarrollo humano.

\section{REFERENCIAS}

Albet, Abel. (2000). Una geografía humana renovada: Lugares y regiones en un mundo global. Barcelona: Vicens Vivens, SA.

Borja, Jordi. (2003). La ciudad conquistada. Madrid: Alianza editorial.

Carrizosa, Umaña Julio. (2003). Colombia, de lo imaginario a lo complejo. Bogotá:
Universidad Nacional de Colombia, Instituto de Estudios Ambientales - IDEA.

Certeau, Michel y otros. (1999). La invención de lo cotidiano. México: Universidad Iberoamericana. Instituto Tecnológico de estudios Superiores de Occidente.

Cuadernos de economía. - Teoría Económica. Disponible en: www.red-vertice.com/fep

Dabas, Elina. Najmanovich, Denise. (2002). Redes. El lenguaje de los vínculos. México: Paidós.

Geerts Clifford. (2001). La interpretación de las culturas. Barcelona: Gedisa, S.A.,

Giraldo Isaza Fabio. (2004). Desarrollo y Hábitat. Bogotá: UN Hábitat-CENAC

Halbwachs, Maurice. La memoria colectiva. Prensas universitarias de Zaragoza.

Jacobs, Jane. (1967). Muerte y vida de las grandes ciudades. Madrid: Ediciones Península.

Lefebvre, Hennry. (1973). El derecho a la ciudad. Barcelona: Editorial Peninsula.

Leff, Enrique. (1998). Saber ambiental: sustentabilidad, racionalidad, complejidad. Buenos Aires: Siglo XX,

Leff, Enrique. (2001). Ecología y capital. México: Siglo XXI Editores.

Morin, Edgar. (2000). "Cultura y Conocimiento", en Watzlawick P. y Krieg P., "El ojo del observador". Barcelona: Gedisa.

Sen Amartya. (2003). Desarrollo y libertad. Colombia: Planeta.

Sen, Amartya. (2004). Foro de economía política. Capital humano y capacidad humana

Zuleta, Estanislao. (1982). Sobre la lectura. Información extraída el 25 de marzo de 2005. Disponible en: http://www. mineducacion.gov.co/cvn/1665/ articles-99018_archivo_pdf.pdf 